\title{
ELECTRON SCATTERING AND THE BAND STRUCTURE OF MIXED CRYSTALS $\mathrm{Hg}_{1-x} \mathrm{Fe}_{x} \mathrm{Se}^{*}$
}

\author{
C. SkIERBISZEWSKI ${ }^{a}$, Z. WILAMOWSKI ${ }^{b}$, T. SUSKI ${ }^{a}$, J. KosSUT $^{b}$ \\ AND B. WITKOWSKA ${ }^{b}$ \\ ${ }^{a}$ High Pressure Research Center, Polish Academy of Sciences \\ Sokołowska 29/37, 01-142 Warszawa, Poland \\ ${ }^{b}$ Institute of Physics, Polish Academy of Sciences \\ Al. Lotników 32/46, 02-668 Warszawa, Poland
}

\begin{abstract}
In this paper the dependence of the band structure and the electron scattering mechanisms on the molar fraction $x$ are studied in $\mathrm{Hg}_{1-x} \mathrm{Fe}_{x} \mathrm{Se}$. The crossover from the zero-gap band to the open band-gap configuration at $x \approx 0.08$ is predicted. We explain the drop of the electron mobility for $x>0.002$ by the alloy scattering mechanism.

PACS numbers: 72.10.Fk, 72.20.Fr, 72.80.Jc
\end{abstract}

\section{Introduction}

$\mathrm{HgSe}: \mathrm{Fe}$ is a suitable material for studies of the effects associated with the spatial correlation of charged centers [1,3-5]. The correlation of the localized charges influences the electron concentration $n_{\mathrm{e}}$ and the electron mobility $\mu$ at low temperatures. The theoretical description of the experimental results for the concentrations of iron $n_{\mathrm{Fe}}$ smaller than $2 \times 10^{19} \mathrm{~cm}^{-3}$ is satisfactory only when the correlations between $\mathrm{Fe}^{+}$centers are taken into account $[2,3]$. On the other hand, the mobility drop observed for $n_{\mathrm{Fe}}>2 \times 10^{19} \mathrm{~cm}^{-3}$ has not yet been explained [6]. Also, the details of the composition dependence of $n_{\mathrm{e}}$ on $x$ are not completely understood, particularly in view of disagreements concerning the energy gap vs. $x$ variation [7]. In this paper we put emphasis on determination of the changes of the energetic positions of the band edges with composition of the crystal. As pointed out by Kossut [8], the same two parameters $V$ and $W$ which describe in the linear approximation the $\Gamma_{6}$ and $\Gamma_{8}$, respectively, band edges variation with $x$ can also be used to estimate the scattering rate of the conduction electrons by chemical disorder (the alloy scattering mechanism). Therefore, the analysis of $n_{\mathrm{e}}$ and $\mu$ can provide an independent information related to the band structure of mixed crystals.

*This work is supported in part by the Committee for Scientific Research under grant 204839101. 


\section{Experimental results and analysis}

We performed measurements of the conductivity and the Hall effect at $T=$ $4.2 \mathrm{~K}$ in seven samples of $\mathrm{HgSe}$ with different amount of Fe. The results, together with the literature data, are shown in Figs. 1 and 2. An initial increase in $n_{\mathrm{e}}$

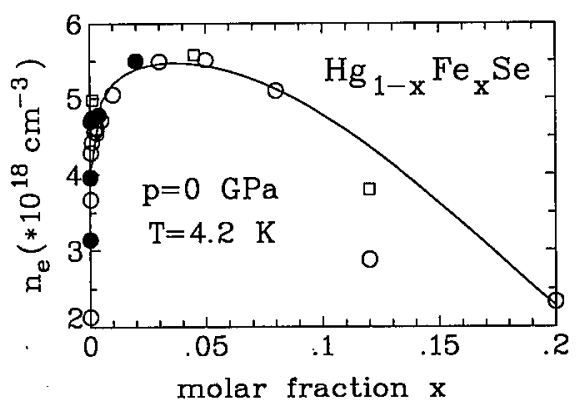

Fig. 1. Electron concentration as a function of molar fraction $x$ in $\mathrm{Hg}_{1-x} \mathrm{Fe}_{x} \mathrm{Se}$ at $T=4.2 \mathrm{~K}$. The experimental results: $\bullet-$ this work; o - Pool et al. [5]; $\square-$ Dobrowolski et al. [4]. Solid lines present the best theoretical fit for $V=3.3 \mathrm{eV}, W=-0.4 \mathrm{eV}$ and $D=0.5 \mathrm{eV}$.

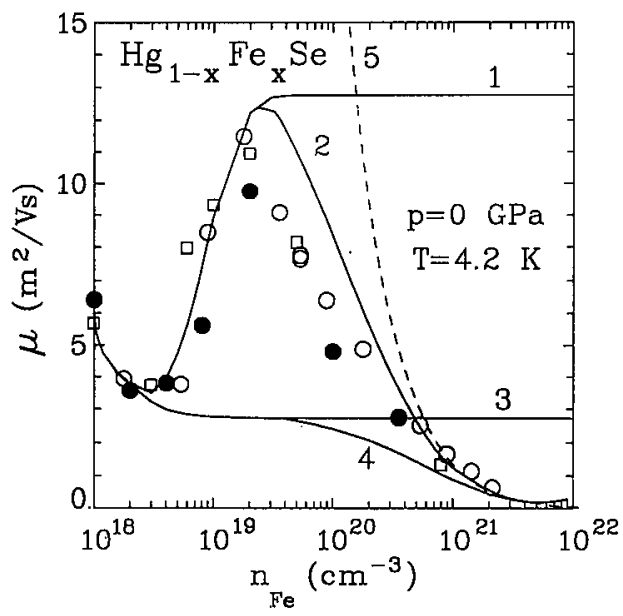

Fig. 2. Electron mobility in $\mathrm{Hg}_{1-x} \mathrm{Fe}_{x} \mathrm{Se}$ samples at $T=4.2 \mathrm{~K}$ as a function of iron concentration. The experimental results: $\bullet-$ this work; $\circ-$ Pool et al. [5]; $\square-$ Dobrowolski et al. [4]. Solid lines present theoretical fits: $1-\mathrm{SRC}$ model, $E_{\mathrm{g}}(x)=E_{\mathrm{g}}^{\mathrm{HgSe}}$; $2-\mathrm{SRC}$ model + alloy scattering, $E_{\mathrm{g}}(x)=E_{\mathrm{g}}^{\mathrm{HgSe}}+3.7 x ; 3$ - ionized impurity scattering for random ion distribution, $E_{\mathrm{g}}(x)=E_{\mathrm{g}}^{\mathrm{HgSe}} ; 4$ - ionized impurity scattering for random ion distribution, $E_{\mathrm{g}}(x)=E_{\mathrm{g}}^{\mathrm{Hg} S}+3.7 x$. Dashed line (5) presents the contribution of the alloy scattering mechanism to the total mobility. 
(Fig. 1) followed by a decrease in its values reflects changes of the energy gap as well as the relative position (with respect to the conduction band edge) and the width of the impurity Fe level. To reproduce experimental data the standard procedure - discussed in detail in [9] - involving the solution of the neutrality equation was extended by introducing an additional parameter $D$ to describe the broadening $\sigma$ of the Fe level. A linear dependence $\sigma(x)=D x$ and a Gaussian shape of this level were assumed. The enhancement of the mobility observed for $n_{\mathrm{Fe}}>4 \times 10^{18} \mathrm{~cm}^{-3}$ (Fig. 2) originates, as mentioned, from the spatial correlations of charged centers $[2,3]$. The effect can be described with sufficient accuracy within the short range correlation model (SRC) - as shown by line labeled 1 in Fig. 2 [3]. For comparison, the electron mobility calculated for random distribution of scattering ions is shown by the line labeled 3 in this figure [10]. Both lines were evaluated neglecting the alloy scattering. Surprisingly, for $n_{\mathrm{Fe}}>2 \times 10^{19} \mathrm{~cm}^{-3}$, in spite of more favorable conditions for the correlation between charged centers to occur, the decrease in the mobility is observed. We will show that this decrease can be interpreted as due to the additional scattering mechanism - the alloy scattering. To describe quantitatively the experimental results we carried out a fitting procedure with 3 fitting parameters: $V, W$ and $D$. We find out, however, that these parameters are not independent. Only the difference $V-W$ can be reliably found from $\mu(x)$ dependence. To evaluate the remaining parameters we assumed additionally that the energetic position of $\mathrm{Fe}$ is constant. With this assumption, which corresponds to Langer-Heinrich ideas [11], we can determine the ratio $V / W$. The best fitting parameters: $V-W=3.7 \pm 0.5 \mathrm{eV} ; V / W=-0.1 \pm 0.1 ; D=0.5 \pm 0.2 \mathrm{eV}$ were obtained in this way. The dashed line in Fig. 2 shows the mobility due to the alloy scattering obtained for values of $V$ and $W$ corresponding to the best fit, namely, $V=3.3 \mathrm{eV}$ and $W=-0.4 \mathrm{eV}$. Lines 2 and 4 show the total mobility taking into account the alloy and ionized impurity scattering for correlated system (SRC theory) and randomly distributed centers, respectively. Let us stress that the alloy scattering depends mainly on the difference $V-W$ and, within the accuracy of determination of $V-W$ of $0.5 \mathrm{eV}$, leads to a reasonable agreement with the experimental data. The difference between the experimental values of the mobility and the theoretical predictions of the SRC theory together with alloy scattering (see line 2 in Fig. 2) for $2 \times 10^{19} \mathrm{~cm}^{-3}<n_{\mathrm{Fe}}<4 \times 10^{20} \mathrm{~cm}^{-3}$ indicates that an additional broadening of the impurity level must occur and that it contributes to an impairment of the spatial correlation in the system of ionized impurities if it exceeds the value of the Coulomb gap. The following conclusions can be drawn from our analysis:

a) the drop of the mobility as a function of $x$ observed at low temperatures for $x>0.002$ is due to chemical disorder (alloy scattering);

b) it is necessary to assume that the Fe related impurity level broadens considerably as $x$ is increased ( $d$-band formation).

c) the crossover from the zero-gap band to the open band-gap configuration takes place at $x \approx 0.08$ and the extrapolated energy gap in hypothetical $\mathrm{FeSe}$ $(x=1)$ is $3.5 \mathrm{eV}$. 


\section{References}

[1] A. Mycielski, P. Dzwonkowski, B. Kowalski, B.A. Orłowski, M. Dobrowolska, M. Arciszewska, W. Dobrowolski, J.M. Baranowski, J. Phys. C 19, 3605 (1986).

[2] J. Mycielski, Solid State Commun. 60, 165 (1986).

[3] Z. Wilamowski, K. Świątek, T. Dietl, J. Kossut, Solid State Commun. 73, 883 (1990).

[4] W. Dobrowolski, K. Dybko, A. Mycielski, J. Mycielski, J. Wróbel, S. Piechuta, M. Palczewska, H. Szymczak, Z. Wilamowski, in: Proc. 18th Int. Conf. Phys. Semicond., Stockholm 1986, Ed. O. Engström, World Scientific, Singapore 1987, p. 1743.

[5] F. Pool, J. Kossut, U. Dębska, R. Reinferberger, Phys. Rev. B 35, 3900 (1987).

[6] I. Kuleev, I. Lyapilin, V. Karyagin, Phys. Status Solidi B 163, 449 (1991).

[7] S.Yu. Paranchich, L.D. Paranchich, V.N. Makogonenko, S.V. Lototskaya, Fiz. Tekh. Poluprovodn. 24, 225 (1990).

[8] J. Kossut, Phys. Status Solidi B 86, 593 (1978).

[9] C. Skierbiszewski, Z. Wilamowski, J. Kossut, in: Proc. 7th Int. Conf. Narrow Gap Semicond., Southampton 1992, Semicond. Sci. Technol., in print.

[10] T. Dietl, W. Szymańska, J. Phys. Chem. Solids 39, 1041 (1978).

. [11] J. Langer, H. Heinrich, in Ref. [4], p. 175. 